\title{
Transport Information Collection Protocol with clustering of information sources
}

\author{
Mohamed Karim SBAÏ \\ Projet Planète, INRIA Sophia Antipolis, France \\ National School of Computer Sciences (ENSI), Tunisia \\ Email: Mohamed_Karim.Sbai@sophia.inria.fr
}

\author{
Chadi BARAKAT \\ Projet Planète, INRIA Sophia Antipolis; France \\ Email: Chadi.Barakat@sophia.inria.fr
}

\begin{abstract}
We improve and validate TICP, our TCP-friendly reliable transport protocol to collect information from a large number of sources spread over the Internet [1]. A collector machine sends probes to information sources that reply by sending back report packets containing their information. TICP adapts the probing rate in a way to avoid implosion at the collector and network congestion. Lost packets are requested again by TICP until they are correctly received. In this work, we add to TICP a mechanism to cluster information sources in order to probe sources behind the same bottleneck together. This ensures a smooth variation of network conditions during the collection session and hence an efficient handling of congestion at the network bottlenecks. This mechanism is based upon the Global Network Positioning (GNP) Internet coordinate system. By running simulations in ns-2 over realistic network topologies, we prove that TICP with clustering of information sources has shorter collection session duration and causes less packet losses in the
\end{abstract}


network than the initial version that probes sources independently of their locations.

\section{Introduction}

Nowadays, collecting information from a large number of network entities has more and more applications. The collected data can be availability of network entities, statistics on hosts and routers, quality of reception in a multicast session, numbering of population, votes, etc. In this work, we improve the Transport Information Collection Protocol (TICP) which we proposed in [1] to collect information entirely from a large set of sources spread over the Internet. A collector machine sends probes to information sources, which send back report packets containing their information. However, some difficulties come into play when designing TICP:

- There is a risk of network congestion due to bandwidth limitation and the large number of sources. Furthermore, all sources are not behind the same bottleneck which makes the congestion control more difficult.

- The collection traffic can be aggressive towards traffic generated by other applications. In particular, it must not penalise concurrent TCP traffic.

- The loss of probes or reports lengthens the duration of the collection session, which urges for an efficient retransmission scheme.

TICP does not only adapt the probing rate as a function of network conditions, but also tries to minimize the collection session duration by deploying an efficient retransmission strategy. Moreover, it shares network resources fairly with concurrent traffic, namely TCP traffic, by adapting its probing rate in a way similar to how TCP does.

The collector in the former version of TICP [1] probes information sources in a random order. We show in this paper that this strategy causes many problems when moving into large networks which results in longer collection sessions, higher loss ratios and out of control traffic. The reason is that only one control at the TICP collector is used to limit the traffic at the several network bottlenecks simultaneously, which is clearly suboptimal given that congestion of one network bottleneck can be hidden by the low utilization of another bottleneck and vice versa. To probe sources behind the same bottleneck together and separately from other bottlenecks, we add to TICP a mechanism to gather information sources into clusters. This mechanism is based on the modelling of the Internet by a twodimension Euclidean space and its decomposition into clusters. We use to this end the Global Network Positioning system (GNP)[4, 5] that provides Internet host coordinates. Our new mechanism makes it possible to traverse sources from the closest cluster to the collector in terms of RTT (Round Trip Time) to the farthest one, which very probably results in sources behind the same bottleneck probed together before the collector moves to neighbouring sources located behind another bottleneck. This is supposed to improve the efficiency of the congestion control and to ensure a smooth variation of its variables in TICP. 
To evaluate the performances of the protocol thus obtained, we ran simulations with the NS-2 simulator [6] over realistic and complex network topologies. These simulations have shown that TICP with the new mechanism of clustering has better performances than without clustering, and that it outperforms other non adaptive data collection solutions.

The paper is organized as follows. In the second section, we describe the main functionalities of TICP. We show in section III that the former version of TICP has many problems and that we need to cluster sources. In the fourth section, we explain our approach of clustering. The section $\mathrm{V}$ discusses simulations results and the last section concludes the paper.

\section{Transport Information Collection Protocol}

TICP [1] is a reliable transport information collection protocol implementing diverse functionalities. We focus here on those related to error recovery and network congestion control.

\section{II.1 Error recovery}

The TICP collector has a list of all information sources. Every source is distinguished by an identifier that can be for example its IP address. Sources whose reports are lost are probed again and a required to retransmit them until they are correctly received by the collector. To make the retransmission of reports in TICP efficient, the collection session is made as a succession of rounds. In the first round, the collector sends request (probe) packets to all sources following their ranking in the list. In a second round, the collector sends requests to sources whose reports were not received in the previous round. The collector continues in rounds until it receives all reports. This behaviour in rounds is meant to wait for transitory network congestion to disappear from one round to another and to absorb the excessive delay that some reports may experience.

\section{II.2 Congestion control}

To control the rate of requests and reports across the network, TICP is based on a report-clocked window based congestion control similar to the TCP one [2]. The collector maintains one variable $c$ wnd indicating the congestion window size in number of requests/reports. New requests are transmitted only when the number of expected reports pipe is less than cwnd. TICP adapts cwnd to the observed loss rate of reports. It proposes two algorithms to do so: Slow start and Congestion Avoidance. 


\section{Slow Start}

The collector starts a collection session by setting $c w n d$ to RS (protocol parameter) and sending $R S$ request packets. After some time, reports start to arrive. Some of these reports come on time, others are delayed. A timely report indicates that the network is not congested and that the collector can continue increasing its congestion window: $c$ wnd $=c w n d+1$. This yields a doubling of the probing rate for every window size of probes. The window continues growing in this way until the network becomes congested. At this point, the collector divides its congestion window by two and enters the congestion avoidance phase. The protocol comes back to slow start whenever a severe congestion appears (to be defined later).

\section{Congestion avoidance}

The congestion avoidance phase represents the steady state of TICP. During this phase, the collector increases slowly cwnd in order to probe the network for more capacity. We aim to a linear increase of the congestion window by $R S$ probes every window size of probes. Thus, upon each timely report, the congestion window is increased by: $c w n d=c w n d+R S / c w n d$. When congestion is detected, cwnd is divided by two and a new congestion avoidance phase is started.

\section{II.3 Congestion detection mechanism}

TICP implements a congestion detection mechanism to compute report loss rates and to decide whether a report is on time, delayed or lost. This mechanism is based upon a timer TO scheduled at the beginning of the session and rescheduled again every time it expires.

\section{Round-trip time estimator}

TICP sets the timer of the mechanism to an estimate of RTT (Round TripTime), using the samples of RTT seen so far. The value of the timer is computed using estimates of the average RTT and of its variance. Let srtt and rttvar be the estimates of the average and the mean deviation of the RTT. Let $r t t$ be the measured round-trip time when a report arrives. The collector updates the estimates and the timer $T O$ in the following way:

$$
\begin{gathered}
r t t v a r=3 / 4 . r t t v a r+1 / 4 .|s r t t-r t t| \\
s r t t=7 / 8 . s r t t+1 / 8 . r t t \\
T O=s r t t+4 . r t t v a r
\end{gathered}
$$

This dynamics and the coefficients it involves are inspired from TCP. TCP maintains an estimate of RTT per couple of source and destination, whereas TICP maintains only one estimate of RTT between the collector and all sources. This estimate is adapted when moving from one source to another. 


\section{Detecting network congestion}

TICP computes the report loss ratio during a time window equal to TO. When the timer is scheduled, the collector saves in the variable torecv the number of reports to be received before the expiration of the timer. Let recv be the number of timely reports received between the scheduling of the timer and its expiration. The collector considers then that torecv - recv reports were lost in the network. Consequently, it estimates the loss ratio to $1-($ recv/torecv). The network is considered congested if the loss ratio exceeds the Congestion Threshold (CT) and severely congested if the loss ratio exceeds a higher threshold $S C T>C T$ called the Severe Congestion Threshold. CT and SCT are two parameters of the protocol. TICP sets them as follows:

$$
\begin{gathered}
C T=\min (0.1, R S / c w n d) \\
S C T=\max (0.9, \text { cwnd }- \text { RS/cwnd })
\end{gathered}
$$

Based on these values, network congestion for TICP means that more than RS reports were lost in a window size of probes, while severe congestion means that less than RS timely reports were received.

\section{Delayed and timely reports}

A timely report is a report received before its deadline. The deadline of a report is given by the timer. A report not received before its deadline is assumed to be lost. If it arrives later than the deadline, it is considered to be delayed.

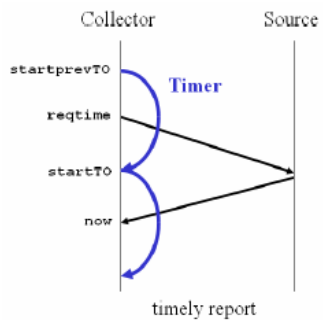

timely report

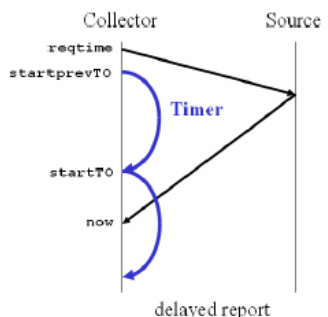

delayed report

Fig.1. The two types of reports

Figure 1 explains how the deadline of a report is set. Let startTO be the scheduling time of the timer. Let startprevTO be the previous scheduling time of the timer. When a report is received, the collector extracts from its header the timestamp reqtime indicating the time by which the corresponding probe has been sent. The report is received on time if and only if startprevTO $<$ reqtime. The report is a delayed one in the opposite case. 


\section{Need for clustering of information sources}

In this section, we present the drawbacks of the former version of TICP that motivated our present work. As we described earlier, the collector has a complete list of sources' identifiers. A collection session is a succession of rounds. In a given round, the collector begins by probing the source at the top of the list, then the following one and so on until the end of the list. The ranking of sources in this list has been so far done randomly and independently of any topology information. In reality, sources are more or less far from the collector. The random ordering results in variable non correlated RTTs during the collection session. Since the estimate of RTT at a given instant depends on its previously measured values, which in the case of random ordering are unrelated, this estimate seldom gives a good idea on the RTT of the next pair probe/report. This causes several problems. First, an overvaluation of RTT results in a delay in the detection of network congestion; the collector waits more than necessary for already lost reports. This delay means a waste of time and an aggravation of network congestion since the probing rate will not be reduced on time. On the other hand, an undervaluation of RTT can cause errors in the computation of report loss rate since the timer expires prematurely. Thus, some reports are declared lost while they are not. In this case, we reduce unnecessarily the size of the congestion window (cwnd) and hence, we increase the collection session duration.

Furthermore with random ranking of sources, packets generated can circulate everywhere in the network. At a given moment, this traffic can participate in the congestion of many bottlenecks. Since it is difficult to adapt congestion window size to network conditions on all paths from sources to collector, the Internet is considered by the original version of TICP as a single bottleneck. This version of TICP does not ensure fairness with concurrent traffic and its mechanism of congestion control is not efficient in case of large networks.

All the drawbacks described above are due to the random ordering by which information sources are probed. It is then important to cluster sources so that those close to each other are probed simultaneously. Also it is important to rank clusters from the nearest to the most distant from the collector so that to ensure that the network conditions vary smoothly and hence TICP congestion control can track them efficiently. The contribution of the present work is the addition to TICP of such a clustering and ranking mechanism together with its validation with extensive simulations.

A cluster is a group of sources located in the same neighbourhood. Our idea is that the more sources are close to each other the more their reports meet the same network conditions on their paths to the collector and the more probable they are located behind the same bottleneck. In this case, the loss of reports indicates that the common bottleneck is congested; hence the collector can handle this congestion efficiently by decreasing the probing rate. The collector probes clusters from the nearest to the farthest. This ensures a smooth variation of the congestion control parameters of TICP, for instance the rate of sending probes and the estimate of RTT. This again results in an efficient network congestion control. 


\section{Clustering of information sources}

In this section, we describe our approach to cluster information sources. For this, we use the Global Network Positioning (GNP) system to model the Internet by a 2-dimensional Euclidean space [4]. A host is represented by a point in this space. The mathematical distance function gives an approximate value of the RTT between any 2 hosts. To ensure this, a small set of hosts called landmarks distributed across the Internet first compute their own coordinates in this geometric space. These coordinates are then disseminated to any ordinary host willing to compute its own coordinates relative to the coordinates of the landmarks [5].

The collector and information sources participate in GNP as ordinary hosts. At the end of the GNP operations, each source has a couple of coordinates $H\left(x_{H}, y_{H}\right)$ and the collector has also its own coordinates $C\left(x_{C}, y_{C}\right)$.

We define a cluster as being a set of information sources whose representing GNP points are located in a square area. The side of the square is denoted $a$, which is a parameter of the protocol. The central cluster is the square whose centre is the point representing the collector $C\left(x_{C}, y_{C}\right)$.

A cluster is completely defined by a couple of coordinates $(X, Y)$ being integer values. These coordinates are those of the centre of the corresponding square relative to the collector coordinates and normalised by $a$. An information source whose coordinates equal to $H\left(x_{H}, y_{H}\right)$ belongs to the cluster $(X, Y)$ given by:

$$
\begin{aligned}
& X=\operatorname{round}\left(\left(x_{H}-x_{C}\right) / a\right) \\
& Y=\operatorname{round}\left(\left(y_{H}-y_{C}\right) / a\right)
\end{aligned}
$$

In order to probe information sources from the nearest to the farthest, the collector begins with the central cluster and then follows a spiral trajectory. Figure 2 gives an idea on this trajectory. One can with a simple algorithm find the coordinates of the next cluster during the collection knowing the coordinates of the current cluster.

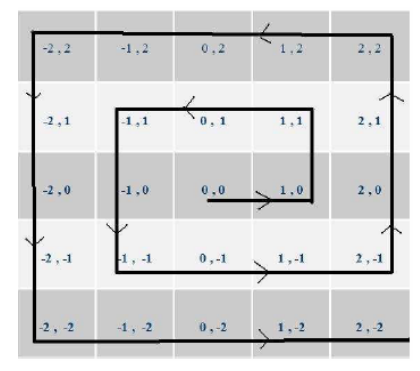

Fig. 2. Order of probing information sources 


\section{Simulation results}

In this section, we discuss the results of our simulations. We have run these simulations in ns-2 [6] in order to evaluate the performance of TICP with and without clustering of information sources. That is why we have implemented GNP and TICP in ns-2.

We generate realistic network topologies for simulations using GT-ITM (Georgia Tech-Internet Topology Modelling) [7, 8]. We choose to work on transit-stub (TS) topologies which give the ability to model the complexity and the hierarchical structure of the real Internet. TS topologies model networks using a 2-level hierarchy of routing domains with transit domains interconnecting lower level stub domains. To these TS topologies, we assign latencies of $35 \mathrm{~ms}$ for intra-transit domain links, $10 \mathrm{~ms}$ for stub-transit links and $5 \mathrm{~ms}$ for intra-stub domain links. Figure 3 gives an example of an TS topology. Table I shows the parameters of the TS topologies used in ours simulations. In each simulation, we choose randomly 500 sources of information and a collector among the nodes that compose each TS topology. The parameters of TICP are set as in Table II.

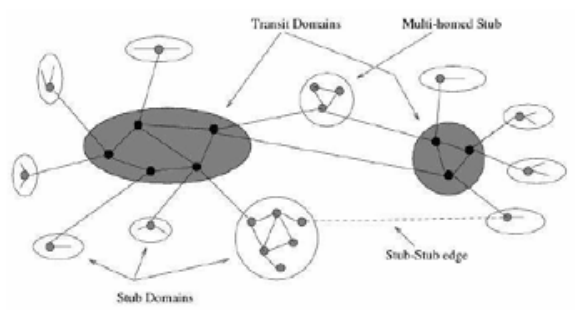

Fig. 3. Transit-Stub topologies

Table I. Transit-stub model parameters

\begin{tabular}{ccc}
\hline Parameter & Signification & Scenario \\
\hline $\mathrm{T}$ & Number of transit domains & 5 \\
$\mathrm{~N}_{\mathrm{t}}$ & Average Number of nodes / transit domain & 7 \\
$\mathrm{~K}$ & Number of stub domains / transit node & 8 \\
$\mathrm{Ns}$ & Average number of nodes / stub domain & 7 \\
\hline
\end{tabular}

Table II. TICP parameters

\begin{tabular}{cc}
\hline Parameter & Value \\
\hline RS & 10 \\
probe size & $100 \mathrm{~b}$ \\
report size & $1500 \mathrm{~b}$ \\
$\mathrm{a}$ & $50 \mathrm{~ms}$ \\
\hline
\end{tabular}




\section{V.1 Network congestion}

We compare between the both versions of TICP with and without clustering of information sources. The comparison criteria are network congestion and duration of the collection session. Figure 4 illustrates an example of the evolution of the congestion window size (cwnd) as a function of time for TICP without clustering. First, we notice the saw tooth behaviour of TICP which adapts the window size to network conditions using the information on the loss ratio. But, we also notice that at time $17 \mathrm{~s}$, there was a reset of cwnd to RS following a severe network congestion (loss rate> SCT). With random probing, TICP is unable to adapt the probing rate to the available bandwidth in several bottlenecks simultaneously. Figure 5 plots the same result but this time for TICP with clustering. It is clear that in this case TICP remains in the congestion avoidance phase and that the severe congestion does not appear. This illustrates that TICP with clustering adapts the window size to its right value without overwhelming the network. One can see TICP with clustering as treating bottlenecks one by one rather than at once. Note in the figures the decreasing trend in the window size, which is the result of the probing of sources from the closest to the collector to the farthest from it. TICP with clustering finishes the collection earlier because there was no congestion. The next paragraph studies the collection session duration.

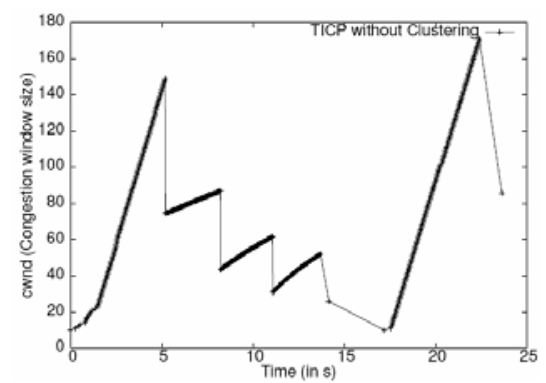

Fig. 4. Cwnd as a function of time for TICP without clustering

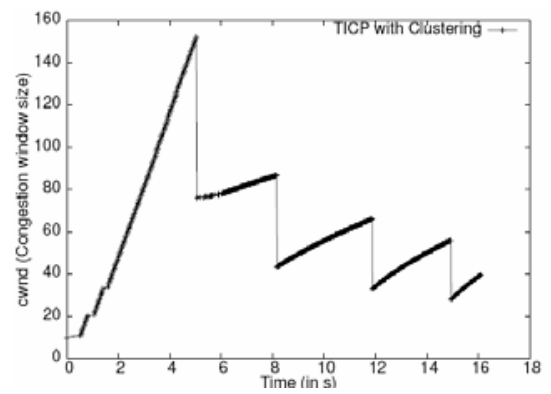

Fig. 5. Cwnd as a function of time for TICP with clustering 


\section{V.2 Collection session duration}

We continue the comparison between the two versions of TICP. This time we concentrate on collection session duration. Figure 6 shows this duration for several simulations of TICP without clustering. In each simulation, the order of sources in the list of the collector is different, that is why we obtain each time different collection session duration. For TICP with clustering, the result is the same since the topology does not change. TICP with clustering finds the good order of information sources and has the shortest collection session duration. We save on average $30 \%$ of the collection session duration by moving from TICP without clustering to TICP with clustering.

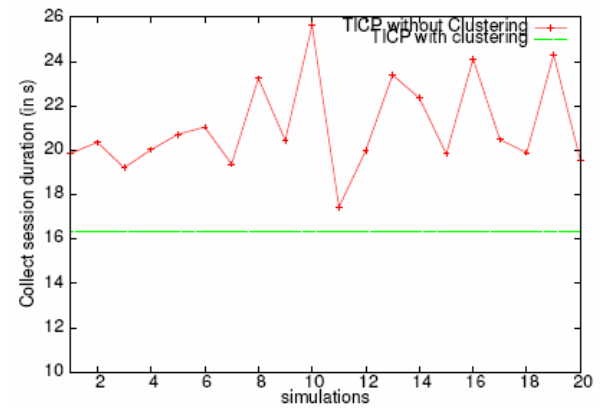

Fig. 6. Collection session duration for different ordering of sources

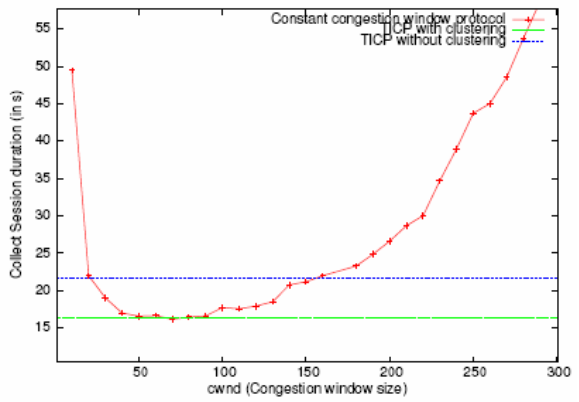

Fig. 7. Optimality of the protocol

To evaluate the optimality of TICP more generally, we implement in ns-2 an information collection protocol having a constant congestion window size. For each window size, we run 10 simulations and we record the minimum of the collection session duration over them. Figure 7 presents the evolution of this duration as a function of cwnd. The curve has a parabolic shape: for small congestion window sizes, collection session duration is long because we have a low probing rate. For large window sizes, the network is congested which lengthens the collection session duration. The role of TICP is to find dichotomicly the good congestion window size that minimizes the collection session duration. We notice in Figure 7 
how TICP with clustering manages to reach the optimum unlike TICP without clustering which yields longer durations.

\section{V.3 Impact of cluster size}

We vary the cluster size and we study its impact on collection session duration. Taking a very large $a$ is equivalent to TICP without clustering since sources will be probed independently of their locations within the large cluster. Taking $a$ very small results in clusters empty or with few number of sources which is not efficient since there will be no clustering of sources behind common bottlenecks. There should be some average $a$ that provides the best performance. Figure 8 validates this intuition where we can see that over the network topologies we considered, a value of $a$ around 50ms is optimal. Each point in the curve of Figure 8 is the average over 5 simulations run on different network realizations satisfying the characteristics in Table I. The number of sources is taken equal to 500 .

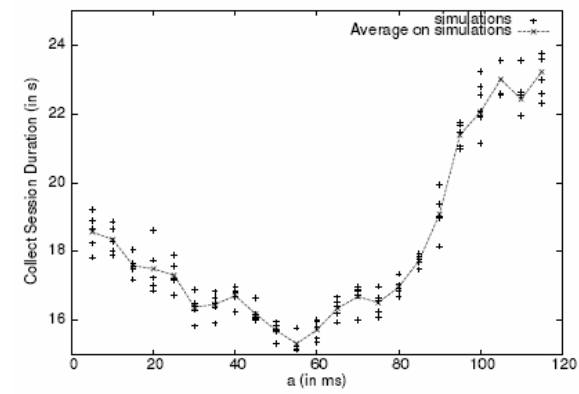

Fig. 8. Impact of $a$ on collection session duration

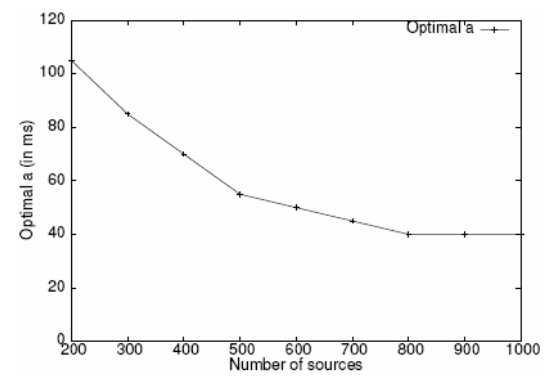

Fig. 9. Optimal $a$ as a function of the number of sources

Figure 9 studies how the number of sources impacts the choice of optimal $a$. We can clearly see that the optimal cluster size decreases when the number of sources increases. Compared to the value used above, the optimal $a$ is equal to $85 \mathrm{~ms}$ for 300 sources and to $45 \mathrm{~ms}$ for 700 sources. Indeed, for small number of sources, one needs to increase $a$ to group more sources behind the same bottleneck to- 
gether. At the opposite, for more sources, one needs to decrease $a$ so that the collector can better probe them depending on their locations. But, if we continue increasing the number of sources, the optimal $a$ will stabilize and become equal to some minimum value depending on the topology. One can safely use this value for applications collecting data from a very large number of sources. We suggest that in reality, one calculates this value by running multiple collections when TICP is used for the first time, then adapts it as a function of the measured session duration to account for any change in the underlying network topology.

\section{Conclusions and perspectives}

TICP is a transport protocol to collect information from a large number of network entities. It aims to control the congestion of the network and to minimize the collection session duration. To ensure a smooth variation of the congestion control parameters, we have added to TICP in this work a mechanism to cluster information sources. The simulation results show that this mechanism ameliorates the performances of TICP. In fact, it reduces loss rate and yields shorter collection session durations. However, the work on TICP is still not yet achieved. Our current research focuses on the implementation of the protocol and on its extension to account for sources of large amounts of data. In this new context, a report will be composed of several packets instead of one packet as it is now.

\section{References}

1. Chadi Barakat, Mohamed Malli, Noamichi Nonaka, "TICP: Transport Information Collection Protocol", in Annals of Telecommunications, vol.61, no. 1-2, pp.167-192, January-February, 2006.

2. M. Allman, V. Paxson, W. Stevens, "TCP Congestion Control", RFC 2581, April.1999.

3. V. Paxson, M. Allman, "Computing TCP's Retransmission Timer", Internet Draft, April 2000.

4. T.S Eugene Ng and Hui Zhang, "Predicting Internet Network Distance with Coordinates-Based Approaches", INFOCOM'02, New York, NY, June 2002.

5. T. S. Eugene Ng and Hui Zhang, "Towards Global Network Positioning", Extended Abstract, ACM SIGCOMM Internet Measurement Workshop, San Francisco, CA, November 2001.

6. The Network Simulator ns-2, http://www.isi.edu/nsnam/ns/

7. Ellen W. Zegura, Ken Calvert and S. Bhattacharjee, "How to Model an Internetwork". Proceedings of IEEE Infocom '96, San Francisco, CA.

8. Ken Calvert, Matt Doar and Ellen W. Zegura, "Modelling Internet Topology”, IEEE Communications Magazine, June 1997. 